Lindner, Karin

\title{
Langfristige Auswirkungen von Auslandspraktika auf die berufliche Entwicklung
}

Haushalt in Bildung \& Forschung 5 (2016) 4, S. 109-122

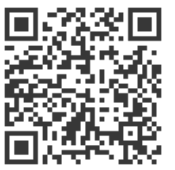

Quellenangabe/ Reference:

Lindner, Karin: Langfristige Auswirkungen von Auslandspraktika auf die berufliche Entwicklung - In:

Haushalt in Bildung \& Forschung 5 (2016) 4, S. 109-122 - URN: urn:nbn:de:0111-pedocs-203436 - DOI: 10.25656/01:20343

https://nbn-resolving.org/urn:nbn:de:0111-pedocs-203436

https://doi.org/10.25656/01:20343

in Kooperation mit / in cooperation with:

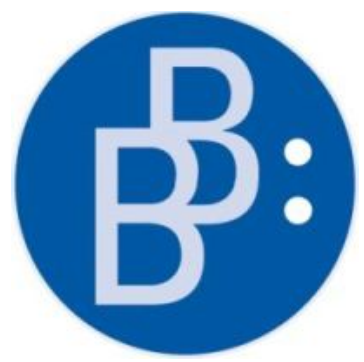

https://www.budrich.de

\section{Nutzungsbedingungen}

Gewährt wird ein nicht exklusives, nicht übertragbares, persönliches und beschränktes Recht auf Nutzung dieses Dokuments. Dieses Dokument ist ausschließlich für den persönlichen, nicht-kommerziellen Gebrauch bestimmt. Die Nutzung stellt keine Übertragung des Eigentumsrechts an diesem Dokument dar und gilt vorbehaltlich der folgenden Einschränkungen: Auf sämtlichen Kopien dieses Dokuments müssen alle Urheberrechtshinweise und sonstigen Hinweise auf gesetzlichen Schutz beibehalten werden. Sie dürfen dieses Dokument nicht in irgendeiner Weise abändern, noch dürfen Sie dieses Dokument für öffentliche oder kommerzielle Zwecke vervielfältigen, öffentlich ausstellen, aufführen, vertreiben oder anderweitig nutzen.

Mit der Verwendung dieses Dokuments erkennen Sie die Nutzungsbedingungen an.

\section{Terms of use}

We grant a non-exclusive, non-transferable, individual and limited right to using this document.

This document is solely intended for your personal, non-commercial use. Use of this document does not include any transfer of property rights and it is conditional to the following limitations: All of the copies of this documents mus retain all copyright information and other information regarding legal protection. You are not allowed to alter this document in any way, to copy it for public or commercial purposes, to exhibit the document in public, to perform, distribute or otherwise use the document in public.

By using this particular document, you accept the above-stated conditions of use.

\section{Kontakt / Contact:}

\section{peDOCS}

DIPF | Leibniz-Institut für Bildungsforschung und Bildungsinformation Informationszentrum (IZ) Bildung

E-Mail:pedocs@dipf.de

Internet: www.pedocs.de

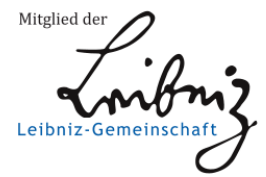




\section{Jahrgang} Heft 4

2016

旁

๓

$\bullet$

号

$\bullet$

돈

용

『্冖

$\frac{\bar{T}}{\Phi}$

$\frac{9}{2}$

정

은

$\stackrel{ \pm}{\frac{1}{2}}$

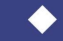

c

$\frac{0}{1}$

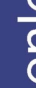

$\xi$

ह

N

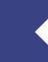

$=$

s

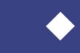

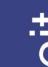

$\frac{2}{2}$

항

ISSN 2193-8806

\section{Haushalt in \\ Forschung}

\section{Lernwirksamkeitsmessung I}

Theoriebildung \& Messinstrumente
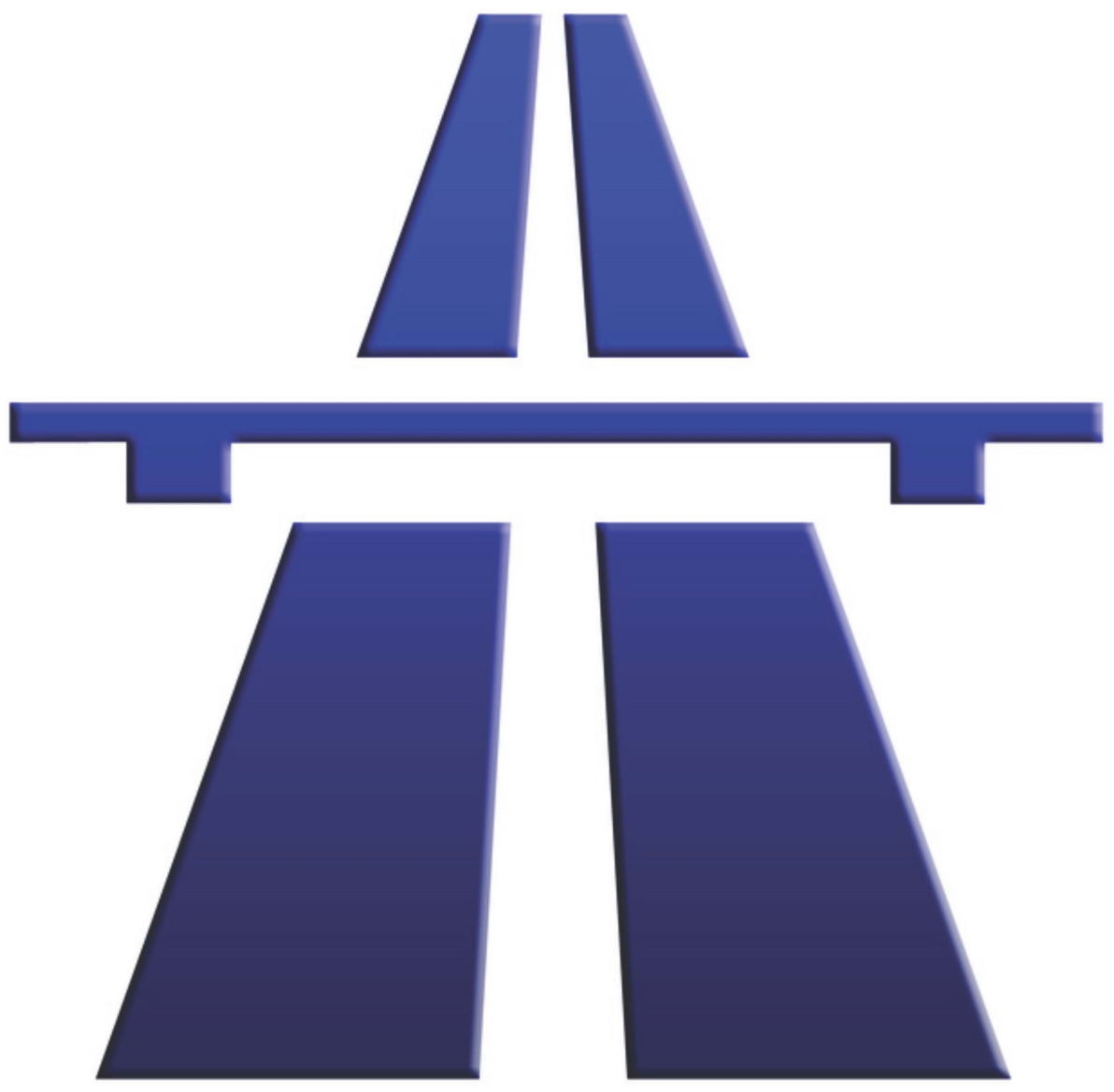


\section{Inhaltsverzeichnis}

Ursula Buchner

Editorial. 2

Werner Brandl

Lernstand erheben, Lernwirksamkeit feststellen,

Lernleistung beurteilen - eine Skizze des Bezugssystems

Gabriele Danninger

Neurodidaktik und Lernwirksamkeit im Lernfeld Ernährung.... 25

Sabine Albert

Die Bedeutung der reflexiven Selbstforschung für die Professionalisierung von Lehrpersonen

Sandro Biaggi \& Claudia Wespi

Professionskompetenzen fördern mit eigenen Videos aus dem Praktikum -

Einblicke in Erfahrungen von Studierenden und Dozierenden

Irmgard Dachtler-Freiler

Neuer Lehrplan - und was nun? Basisüberlegungen zur Lernwirksamkeit des neuen Lehrplans 61

Martina Überall, Maria Lerchbaumer, Christa Meliss \& Birgit Wild

Nachhaltigkeit schmeckt!

Ursula Buchner \& Maria Magdalena Fritz

Lernen im Fach Gesundheit und Soziales: Was wirkt?

Karin Lindner

Langfristige Auswirkungen von Auslandspraktika

auf die berufliche Entwicklung.

Julia Hirsch

Rezension: Essen und Ernährungsbildung in der KiTa 123 
Berufliche Auswirkungen von Auslandspraktika

Karin Lindner

\section{Langfristige Auswirkungen von Auslandspraktika auf die berufliche Entwicklung}

In Österreich stellen in Berufsbildenden Schulen ${ }^{1}$ die als Pflichtpraktika durchgeführten Betriebspraktika für Jugendliche die Möglichkeit, in der Schule vermitteltes Wissen und fachpraktisches Können mit Tätigkeiten in relevanten betrieblichen Situationen zu verknüpfen. Die Studie untersucht, ob ein im Rahmen eines Pflichtpraktikums an einer $\mathrm{HLW}^{2}$ oder HLT ${ }^{3}$ absolviertes Auslandspraktikum langfristige Auswirkungen auf die berufliche Entwicklung hat.

Schlüsselwörter: Auslandspraktika, berufsbildende Schulen, langfristige Auswirkungen, berufliche Entwicklung

\section{Einleitung}

In Österreich bieten in berufsbildenden Schulen Betriebspraktika als Pflichtpraktika die Möglichkeit, schulisches Wissen und Können in der betrieblichen Praxis anzuwenden. Betriebspraktika stellen somit eine Ergänzung für die theoretische und praktische Ausbildung in der Schule dar. Das in der Schule vermittelte theoretische Wissen und das fachpraktische Können werden mit Tätigkeiten in relevanten betrieblichen Situationen verknüpft und in soziale Interaktionen eingebunden. Es findet ein Transfer von Wissen und Können auf konkrete Anwendungssituationen in der Praxis statt. Die Schülerinnen und Schüler sammeln so praktische Erfahrungen und entwickeln sich individuell weiter. Laut Lehrplan kann das Pflichtpraktikum auch im Ausland durchgeführt werden und wird im Hinblick auf fremdsprachliche Kompetenzen auch empfohlen (Bundesministerium für Bildung, o.J.).

$\mathrm{Zu}$ den Aufgaben der Koordinatorin bzw. des Koordinators für Mobilitätsprojekte im Rahmen von Erasmus $+{ }^{4}$ gehört es, im Antrag unter anderem die Projektziele, die zu erwartenden Lernergebnisse sowie die erwartete Wirkung auf die Teilnehmerinnen und Teilnehmer zu beschreiben. Die Erreichung der Ziele, Ergebnisse und Auswirkungen auf die Teilnehmerinnen und Teilnehmer wird im Anschluss an das Projekt evaluiert, anhand von Teilnehmerberichten festgestellt und in den Mobilitätsnachweisen sowie in einem Schlussbericht, der der Nationalagentur für Lebenslanges Lernen ${ }^{5}$ übermittelt wird, dokumentiert. Nach Abschluss des Projekts werden in der Regel keine weiteren Evaluierungen oder Dokumentationen erstellt (Erasmus+, o. J.). 


\section{Berufliche Auswirkungen von Auslandspraktika}

In der Studie wird untersucht, ob Auslandspraktika für Absolventinnen und Absolventen von Höheren Lehranstalten für wirtschaftliche Berufe und Höheren Lehranstalten für Tourismus konkrete langfristige positive Auswirkungen haben, wie etwa ...

- eine erhöhte Beschäftigungsfähigkeit und bessere Aufstiegschancen,

- aktivere Selbstbestimmung und gesteigertes Selbstwertgefühl sowie aktive Teilnahme am gesellschaftlichen Miteinander in Europa,

- erweiterte Fremdsprachenkompetenz und interkulturelles Bewusstsein,

- $\quad$ eine größere Motivation für zukünftige Auslands-Mobilitäten.

\subsection{Problemstellung und Motivation}

In Österreich absolvieren vor allem Schülerinnen und Schüler von Höheren Lehranstalten für wirtschaftliche Berufe und von Höheren Lehranstalten für Tourismus ihr Pflichtpraktikum im Ausland.

Die Erreichung bzw. Nichterreichung der erwarteten Lernergebnisse wird sofort nach dem Auslandsaufenthalt in den Teilnehmerberichten im Rahmen von Erasmus+ Förderprojekten sowie in den mündlichen Rückmeldungen der Schülerinnen und Schüler sichtbar. Diese werden im Projekt-Schlussbericht zusammengefasst und dokumentiert und der Nationalagentur übermittelt. Nun stellt sich die Verfasserin die Frage, ob ein Auslandspraktikum für die Absolventinnen und Absolventen einer HLW bzw. HLT langfristige positive Auswirkungen auf die berufliche Entwicklung hat.

\subsection{Forschungsfeld, Forschungsidee und Forschungsinteresse}

Das Forschungsfeld „Berufs- und Laufbahnforschung“, in dessen Kontext die Studie einzuordnen ist, thematisiert Fragen der Berufswahl, der beruflichen Entwicklung, der Entwicklung beruflicher Interessen im Ausbildungs- und Berufsverlauf sowie der Beziehungen zwischen Persönlichkeit und beruflichen Interessen (JKU Linz, o. J.). Die Studie hinterfragt die Zusammenhänge zwischen Auslandspraktikum und beruflicher Entwicklung.

Die Forschungsergebnisse werden der Nationalagentur Österreich sowie der Europäischen Kommission zur Verfügung gestellt und werden zur Entwicklung von Beratungsangeboten für Schulen und antragstellende Institutionen für Mobilitätsprojekte im Bereich Berufsbildung von Erasmus+ genutzt. Die Bedeutung der Forschungsidee bzw. das Forschungsinteresse liegen darin, dass eine durch die empirische Untersuchung bestätigte langfristige positive Auswirkung auf die berufliche Entwicklung eine größere Motivation für Auslandspraktika darstellt und so die Auslands-Mobilität von Jugendlichen in Österreich weiter gesteigert werden kann. 


\section{Berufliche Auswirkungen von Auslandspraktika}

\subsection{Stand der wissenschaftlichen Forschung}

Zur Frage über langfristige Auswirkungen von Auslandsmobilitäten gibt es mehrere internationale Studien, aber keine Studie, die sich speziell auf Absolventinnen und Absolventen der berufsbildenden Schulen HLW und HLT in Österreich bezieht.

Die Studie „The Erasmus Impact Study“ untersucht unter anderem die Auswirkungen von Auslandsmobilitäten auf die Beschäftigungsfähigkeit ${ }^{6}$ (Employability) von Jugendlichen in acht europäischen Ländern, Österreich ausgenommen. Aus der Studie geht hervor, dass Absolventinnen und Absolventen von Auslandsmobilitäten ihre Beschäftigungsfähigkeit durchschnittlich um $42 \%$ steigern. Internationale Erfahrungen sind den Unternehmen bei der Anstellung von Mitarbeiterinnen und Mitarbeitern sehr wichtig. 93\% der Absolventinnen und Absolventen von Auslandsmobilitäten können sich vorstellen zukünftig im Ausland zu leben. Im Vergleich dazu können sich nur 73\% derjenigen, die in Österreich blieben, vorstellen zukünftig im Ausland zu leben (European Union, 2014, S. 14).

Die Studie „The Professional Value of Erasmus Mobility“, kurz VALERAStudie, untersucht die Auswirkungen von Auslandsmobilitäten und ihre Beziehung zur Beschäftigungsfähigkeit von Jugendlichen. Die Studie bestätigt, dass Absolventinnen und Absolventen von Erasmus+ Mobilititäten ihre internationalen Kompetenzen viel höher einschätzen als Personen, die keine Auslandsmobilität absolviert haben (Janson, Schomburg \& Teichler, 2009).

Die Studie in Großbritannien „Student perspectives on going international“ belegt, dass Auslandsmobilitäten die Beschäftigungsfähigkeit signifikant erhöhen (Jones, 2012).

\section{Das Auslandspraktikum im Rahmen des Pflichtpraktikums}

Im Folgenden werden die Rahmenbedingungen für das Pflichtpraktikum an Höheren Lehranstalten für wirtschaftliche Berufe sowie an Höheren Lehranstalten für Tourismus erläutert und es wird auf die speziellen Anforderungen für ein Auslandspraktikum in diesen Schultypen eingegangen. Einleitend dazu wird zuerst ein kurzer Einblick in das allgemeine Bildungsziel dieser Schultypen gegeben.

\subsection{Allgemeines Bildungsziel an HLW und HLT}

Das allgemeine Bildungsziel in den Lehrplänen dieser beiden Schultypen legt fest, dass die Absolventinnen und Absolventen über jene Kompetenzen verfügen, die sie zur Ausübung von Berufen in dem Schultyp entsprechenden Bereichen befähigt. Die Jugendlichen sollen unter anderem zur aktiven Teilnahme an der Gesellschaft (Acti- 


\section{| Berufliche Auswirkungen von Auslandspraktika}

ve Citizenship) und zur Bereitschaft zu lebenslangem Lernen (Lifelong Learning) befähigt werden. Die Beschäftigungsfähigkeit ist ein wesentliches Ziel vollzeitlicher berufsbildender höherer Schulen und beruht auf beruflicher Handlungskompetenz (Bundesministerium für Bildung). Schwadorf definiert diese in Anlehnung an Bader (2000) als „Fähigkeit und Bereitschaft, in beruflichen Situationen sachgerecht, gruppen- und beziehungsorientiert sowie verantwortlich reflektiert zu handeln" (Schwadorf, 2003, S. 39). Gruber (1999) geht davon aus, dass Erfahrung die Grundlage der beruflichen Handlungskompetenz darstellt. Erfahrung und das praktische Ausüben von Tätigkeiten in komplexen Handlungsbereichen sind Voraussetzung für erfolgreiches Handeln (S. 11).

In der Studie wird in diesem Zusammenhang untersucht, ob Auslandspraktika mit speziellen gewonnenen Erfahrungen die spätere Beschäftigungsfähigkeit verbessern. Die Studie soll außerdem einen Aufschluss darüber geben, ob Auslandspraktika einen Einfluss auf die berufliche Entwicklung der Personen haben.

\subsection{Pflichtpraktika an HLW und HLT}

Laut Bildungs- und Lehraufgabe in den entsprechenden Lehrplänen sollen die Schülerinnen und Schüler während des Pflichtpraktikums ...

- ergänzend zu den in der Ausbildung erworbenen Kenntnissen und Fertigkeiten in einem facheinschlägigen Betrieb (vorzugsweise in einem dem Schultyp entsprechenden Betrieb) jene Gewandtheit der Berufsausübung vertiefen, die den Anforderungen des jeweiligen Berufsfeldes an Absolventinnen und Absolventen der Schulart entspricht;

- die in der Schule erworbenen Sachkompetenzen in der Berufsrealität umsetzen;

- einen umfassenden Einblick in die Organisation von Unternehmen erhalten;

- über Pflichten und Rechte der Arbeitnehmerinnen und Arbeitnehmer Bescheid wissen, um die unmittelbare berufliche Situation daraufhin überprüfen können;

- in der Lage sein, sich Vorgesetzten sowie Mitarbeiterinnen und Mitarbeitern gegenüber freundlich, korrekt und selbstsicher zu präsentieren;

- aus der Zusammenschau der Unterrichts- und Praxiserfahrung eine positive Grundhaltung zum Arbeitsleben insgesamt und zum konkreten beruflichen Umfeld im Besonderen erwerben (Bundesministerium für Bildung, o. J.). 


\section{Berufliche Auswirkungen von Auslandspraktika}

\subsubsection{Arbeitsrechtsrechtliche und sozialrechtliche Bestimmungen für Auslandspraktika}

Auslandspraktika werden von den Schulen grundsätzlich gefördert und unterstützt. Vor Beginn des Praktikums sind die Schülerinnen und Schüler über ihre Rechte und Pflichten zu informieren. Ein Auslandspraktikum sollte auf jeden Fall vorher mit der Schule abgesprochen werden, auch um abzuklären, ob das Praktikum im Ausland anerkannt wird.

Grundsätzlich gelten die arbeits- und sozialrechtlichen Bestimmungen jenes Landes, in dem das Praktikum absolviert wird. Die Entlohnung erfolgt nach den Tarifverträgen des Aufnahmelandes und kann sehr unterschiedlich sein, auch die Lohnabzüge sind meist sehr unterschiedlich. Zwischen Arbeitgeberin bzw. Arbeitgeber und Praktikantin bzw. Praktikant ist die Regelung bezüglich Kost und Quartier zu vereinbaren (Arbeiterkammer, 2014).

\subsubsection{Praktikumsvertrag und Lernvereinbarung}

Die Kontaktaufnahme zu den ausländischen Betrieben erfolgt in der Regel durch die Schülerinnen und Schüler selbst, meist über eine schriftliche Bewerbung, manchmal folgt auch ein persönliches Bewerbungsgespräch über Skype. In einem schriftlichen Arbeitsvertrag (Praktikumsvertrag) werden die genauen Bedingungen, unter denen das Arbeitsverhältnis eingegangen wird, festgelegt:

- Name und Anschrift der Arbeitgeberin bzw. des Arbeitgebers

- Name und Anschrift der Arbeitnehmerin bzw. des Arbeitnehmers

- Beginn, Ende bzw. genaue Dauer des Praktikums

- Dauer der Kündigungsfrist und Kündigungstermin

- genauer Arbeitsort

- Einstufung in ein Schema, vorgesehene Verwendung, Grundgehalt und weitere Entgeltbestandteile, Regelung der Kosten für Unterkunft und Verpflegung, Fälligkeit des Entgelts

- Urlaubsausmaß

- vereinbarte tägliche und wöchentliche Arbeitszeit

- Kollektivvertragszugehörigkeit und Hinweis, in welchem Raum dieser aufliegt

- Name und Anschrift der Mitarbeitervorsorgekasse (Arbeiterkammer, 2014)

Ergänzend zum Praktikumsvertrag, in dem vor allem die rechtlichen Rahmenbedingungen festgelegt werden, soll bei Auslandspraktika zwischen Praktikantin bzw. Praktikant und Arbeitgeberin bzw. Arbeitgeber eine Lernvereinbarung (Learning Agreement) abgeschlossen werden. Von $\mathrm{ECVET}^{7}$ wurde ein Formular entwickelt, 


\section{| Berufliche Auswirkungen von Auslandspraktika}

das als Vorlage für ein Learning Agreement zur Verfügung steht und an den schuleigenen Bedarf angepasst werden kann (ECVET projects, 2013). Das Learning Agreement stellt einen Kontrakt für die Zusammenarbeit zwischen Entsendeeinrichtung (Schule), Aufnahmeeinrichtung (Praktikumsbetrieb) und Praktikant bzw. Praktikant dar. Im Learning Agreement werden von der entsendenden Schule folgende Punkte dokumentiert:

- Kenntnisse, Fertigkeiten und Kompetenzen, die die Praktikantin bzw. der Praktikant aufgrund des aktuellen Ausbildungsstands vorweisen kann.

- Aufgaben, die die Praktikantin bzw. der Praktikant während des Praktikums nach einer Einschulungsphase übernehmen und selbstständig durchführen kann.

- Die zu erwartenden Lernergebnisse - jene fachlichen, sozialen, sprachlichen sowie interkulturellen Kompetenzen, die die Praktikantin bzw. der Praktikant während des Praktikums erwerben soll.

So erhält die Arbeitgeberin bzw. der Arbeitgeber einen Überblick über das aktuelle fachliche und sprachliche Kompetenzprofil der Praktikantin bzw. des Praktikanten. Von der Aufnahmeeinrichtung werden anschließend folgende Punkte im Learning Agreement ergänzt:

- Aufgaben und Tätigkeiten, die die Praktikantin bzw. der Praktikant während des Praktikums tatsächlich übernehmen wird.

- Die verwendeten Sprachen.

In der Lernvereinbarung wird ebenfalls vereinbart, wer im Rahmen des Praktikums welche Aufgaben übernimmt (z. B.: Monitoring, Mentoring, Evaluierung des Praktikums, Dokumentation, Validierung etc.). Durch ein konkretes Agreement wird im Vorfeld die Intensität und Qualität der Praktikumsbetreuung durch die Arbeitgeberin bzw. den Arbeitgeber geklärt. Die persönliche Betreuung der Praktikantinnen bzw. Praktikanten im Betrieb ist für ein erfolgreiches Auslandspraktikum besonders wichtig und reicht von Einführung, Beratung, Begleitung, Vermittlung von Fachwissen bis hin zur Hilfestellung bei Problemen. Im Learning Agreement kann auch angeführt werden, wer die schulische Ansprechperson für das Unternehmen ist, falls Probleme auftauchen.

Die Lernvereinbarung als ECVET-Element fördert die Umsetzung von ECVET in Österreich hinsichtlich einer erhöhten Durchlässigkeit innerhalb des nationalen Bildungssystems durch verbesserte gegenseitige Anerkennung erbrachter Lernleistungen innerhalb Europas sowie eine verbesserte Anerkennung von formal und informell erworbenen Lernergebnissen. Durch die Verwendung von ECVET Elementen wird eine Qualitätssteigerung der Auslandspraktika und durch die Dokumentation der erworbenen Kompetenzen eine erhöhte Beschäftigungsfähigkeit auf dem österreichischen und europäischen Arbeitsmarkt ermöglicht (ECVET, o. J.). 


\section{Berufliche Auswirkungen von Auslandspraktika}

\subsection{Rahmenbedingungen und Ziele für Auslandspraktika}

Im folgenden Kapitel werden Rahmenbedingungen und Ziele für Auslandspraktika erläutert.

\subsubsection{Betreuung durch die Schule}

Die Schule hat laut Lehrplan (Bundesministerium für Bildung) beim Auffinden einer geeigneten Praktikumsstelle Hilfestellung anzubieten, ist jedoch nicht dafür verantwortlich, dass solche in ausreichender Anzahl zur Verfügung stehen. Das Pflichtpraktikum ist je nach Schultyp in den entsprechenden Unterrichtsgegenständen vorzubereiten und die Lernenden sind hinsichtlich der Betriebskategorie und der Einsatzbereiche zu beraten. Die Erfahrung hat gezeigt, dass Schülerinnen und Schüler mit verschiedenen Erwartungen und Bedürfnissen in die Praktikumsbetriebe kommen - und auch die Arbeitgeberinnen und Arbeitgeber unterschiedliche Anforderungen stellen. Wesentlich für ein Praktikum mit großem Lernerfolg ist daher, den Schülerinnen und Schülern im Vorfeld zu vermitteln, welche individuellen Eigenschaften und Voraussetzungen für einen bestimmten Betrieb notwendig sind.

Die Schule soll laut Lehrplan darauf hinwirken, dass beim Abschluss von Praktikumsverträgen die relevanten arbeits- und sozialrechtlichen Bestimmungen eingehalten werden. Es ist an den meisten Schulen üblich, dass rechtzeitig vor Beginn des Pflichtpraktikums ein Informationsabend für Praktikantinnen und Praktikanten und deren Erziehungsberechtigte abgehalten wird.

Laut Lehrplan sind die Lernenden von der Schule zu veranlassen, in geeigneter Weise Aufzeichnungen über ihre Arbeitszeiten und ihre Tätigkeiten während ihres Praktikums zu führen, die in den facheinschlägigen Unterrichtsgegenständen des folgenden Semesters ausgewertet werden können. Für diese Aufzeichnungen kann von der Schule ein Arbeitstagebuch oder Praxistagebuch zum Ausfüllen vorgegeben werden.

Am Ende des Praktikums ist die Arbeitgeberin bzw. der Arbeitgeber verpflichtet, ein Praktikumszeugnis auszustellen. Dieses dient der formalen Anerkennung des Praktikums in der Schule. Empfehlenswert ist zusätzlich die Ausstellung eines Europass Mobilitätsnachweises. Dieser dokumentiert Lernfortschritte sowohl fachlicher Kompetenzen, als auch nicht formal erworbener Fähigkeiten, kann online erstellt und späteren Bewerbungen beigelegt werden (NEC Österreich, o. J.).

Laut Lehrplan ist das Pflichtpraktikum in den entsprechenden Unterrichtsgegenständen ausführlich nachzubereiten und zu evaluieren. Die Reflexion des Praktikums ist für einen erfolgreichen Lernprozess unbedingt erforderlich. Drees und Pätzold beschreiben die Praxisreflexion als analysierendes, systematisierendes, kritisch abwägendes, vertiefendes und perspektivenbildendes Nachdenken über die Erfahrungen während des Praktikums. Eine gezielte Reflexion des Praktikums soll im Unter- 


\section{Berufliche Auswirkungen von Auslandspraktika}

richt in der Schule initiiert werden, um zusätzliche Lerneffekte zu ermöglichen (Drees \& Pätzold, 2006, S. 353ff).

Ein Portfolio kann die Lernprozesse während des Praktikums sichtbar machen, indem Lernentwicklungen abgebildet, individuelle Lernzuwächse aufgezeigt und Lernprodukte dokumentiert werden. Der individuelle Lernprozess wird reflektiert und Lernergebnisse dokumentiert (Endres, Wiedenhorn \& Engel, 2008, S. 208f). Dazu ist es notwendig, im Vorfeld das Portfolio mit den Schülerinnen und Schülern zu besprechen und erforderliche Inhalte und die Form der Arbeit festzulegen.

\subsubsection{Sprache}

Meist wählen Schülerinnen und Schüler ein Land für ihr Praktikum, in dem die Arbeitssprache eine der Fremdsprachen ist, die sie in der Schule lernen. Die Schülerinnen und Schüler werden nur zum Teil im Fremdsprachenunterricht konkret auf ihr Praktikum und die Kommunikation während des Praktikums vorbereitet. In manchen Schulen werden spezielle Fremdsprachen-Workshops für die Auslandspraktikantinnen und -praktikanten angeboten, in denen gezielt Vokabeln und Redewendungen trainiert werden, die sie für die Tätigkeiten und Konversationen in Hotellerie und Gastronomie benötigen.

Nimmt eine Schule am Erasmus+ Mobilitätsprojekt teil, müssen die Schülerinnen und Schüler vor Beginn des Auslandsaufenthaltes über die Plattform Erasmus+ OLS (Online Linguistic Support) einen Online-Sprachkurs in jener Fremdsprache absolvieren, die Arbeitssprache während des Praktikums ist. Sie erhalten als Feedback eine Einstufung ihrer Kenntnisse und Kompetenzen. Während des Auslandsaufenthalts können die Schülerinnen und Schüler einen Online-Sprachkurs absolvieren und nach Abschluss des Auslandsaufenthalts durch einen zweiten Online-Test die Verbesserung ihrer Sprachkenntnisse feststellen.

In der Studie wird ermittelt, ob ein Auslandspraktikum auch langfristig positive Auswirkung auf die Verwendung von Fremdsprachen bzw. in Zusammenhang mit der Fremdsprache auf die Employability der Personen hat. Für die Studie werden Personen befragt, die ein Praktikum in einem fremdsprachigen Land absolviert haben, die Arbeitssprache muss Englisch, Französisch, Spanisch oder Italienisch gewesen sein.

\subsubsection{Interkulturelle Kompetenz}

Interkulturelle Kompetenz ist ein sehr komplexer Begriff und erstreckt sich über die drei Kompetenzdimensionen Selbst-, Sach- und Sozialkompetenz bzw. baut auf diese auf (Loiselle, 1999, S. 439). 


\section{Berufliche Auswirkungen von Auslandspraktika}

Die „Erasmus Impact Studie“ zeigt auf, dass Absolventinnen und Absolventen von Auslandspraktika davon überzeugt sind, aus einer Auswahl von Fähigkeiten folgende fünf am meisten verbessert zu haben:

- in der Lage sein, mit Menschen anderer Herkunft und Kulturen zu interagieren und $\mathrm{zu}$ arbeiten

- $\quad$ sich in neuen Situationen anpassen und handeln

- Kenntnis von Kultur, Gesellschaft und Wirtschaft des Landes

- Interkulturelle Kompetenzen

- Fremdsprachenkompetenz (European Union, 2014, S. 111).

67\% der für die „Erasmus Impact Studie“ befragten Absolventinnen und Absolventen eines Auslandspraktikums geben an, dass Interkulturelle Kompetenzen für die Arbeitgeberin bzw. den Arbeitgeber für das Recruiting für die erste Arbeitsstelle nach Abschluss der Ausbildung sehr wichtig war (European Union, 2014, S. 116). 83\% der befragten Absolventinnen und Absolventen geben an, dass die Fähigkeit mit Personen anderer Kulturen zusammen zu arbeiten eine wichtige Fähigkeit für eine erfolgreiche Karriere ist (European Union, 2014, S. 104). 69\% der befragten Mobilitäts-Absolventinnen und Absolventen geben an, dass ihre derzeitige berufliche Tätigkeit Merkmale der Internationalisierung aufweist (European Union, 2014, S. 117). $52 \%$ geben noch fünf bzw. zehn Jahre nach ihrer Auslandsmobilität an, dass sie internationale Geschäftskontakte pflegen (European Union, 2014, S. 120). Diese internationale Orientierung von Tätigkeiten der Absolventinnen und Absolventen von Auslandsmobilitäten zeigt sich auch in der Verwendung der Fremdsprachen (European Union, 2014, S. 121).

In dieser Studie wird untersucht, ob die Ergebnisse der „Erasmus Impact Studie“ sich mit den Ergebnissen in Österreich decken.

\section{Planung, Ablauf und Methodik der Studie}

Die Organisation der Planung und des Ablaufs der geplanten Studie richten sich nach den Phasen einer empirischen Untersuchung von Diekmann (2016, S. 187-197).

\subsection{Formulierung und Präzisierung des Forschungsproblems}

Die Forschungsfrage der Arbeit lautet nach Darstellung des Themas und der Problemlage folgendermaßen:

Welche Auswirkungen hat ein im Rahmen des Pflichtpraktikums an einer HLW oder HLT absolviertes Auslandspraktikum langfristig auf die berufliche Entwicklung?

Sieht man von der übergeordneten Forschungsfrage ab, so ergeben sich noch zahlreiche Unterfragen, die im Verlauf der Studie erläutert und beantwortet werden 


\section{| Berufliche Auswirkungen von Auslandspraktika}

sollen. Vor allem muss geklärt werden, woran man erkennen kann, welche langfristigen Auswirkungen ein Auslandspraktikum auf die berufliche Entwicklung einer Person hat und welche konkreten Faktoren Aufschluss darüber geben, ob ein absolviertes Auslandspraktikum langfristig positive Auswirkungen auf die berufliche Entwicklung hat. Dazu wurden folgende Hypothesen $(\mathrm{H})$ formuliert:

Wenn eine Person während ihrer Schulzeit an einer HLW oder HLT ein Auslandspraktikum absolviert hat, ...

H 1: ... hat sie gegenüber anderen Personen Vorteile bei einer beruflichen Anstellung nach Abschluss der Schule.

H 2: ... hat sie berufliche Vorteile aufgrund der besseren Fremdsprachenkenntnisse.

H 3: ... ist sie bereit im Rahmen ihrer beruflichen Tätigkeit ins Ausland zu gehen.

H 4: ... ist sie anderen Kulturen gegenüber aufgeschlossen.

Im Folgenden werden die in den Hypothesen auftretenden Begriffe definiert und operationalisiert:

Die für die Studie untersuchte Personengruppe hat eine Höhere Lehranstalt für wirtschaftliche Berufe oder eine Höhere Lehranstalt für Tourismus besucht und ihr Pflichtpraktikum in einem Betrieb der Gastronomie oder Hotellerie im fremdsprachigen Ausland absolviert. Die Arbeitssprache während des Praktikums war Englisch, Französisch, Spanisch oder Italienisch. Der Auslandsaufenthalt hat mindestens acht Wochen gedauert. Die Personengruppe hat die Schulausbildung vor mindestens drei Jahren erfolgreich abgeschlossen und ist seit mindestens einem halben Jahr in einem Betrieb angestellt. Die Vorteile bei einer beruflichen Anstellung können sich daraus ergeben haben, dass die Person aus einer Gruppe von Bewerberinnen und Bewerbern aufgrund ihres Auslandspraktikums ausgewählt wurde. Hier könnte sich als Problem ergeben, dass die Person das nicht weiß, falls dies nicht beim Bewerbungsgespräch oder später thematisiert wurde.

Die Bereitschaft der Person, während ihrer beruflichen Tätigkeit ins Ausland zu gehen, kann sowohl im Fragebogen abgefragt werden, als auch im Interview detaillierter hinterfragt werden.

Die beruflichen Vorteile der Person aufgrund besserer Fremdsprachenkenntnisse können durch den Einsatz und die Verwendung der Fremdsprache eruiert werden. Wenn die Person im Unternehmen für Korrespondenz oder Kommunikation in der Fremdsprache eingesetzt wird, hat sie entsprechende berufliche Vorteile durch die besseren Fremdsprachenkenntnisse.

\subsection{Datenerhebung und Datenauswertung}

Zur Beantwortung der Forschungsfrage werden in der Studie sowohl quantitative als auch qualitative Methoden angewendet. Die Sampling-Gruppe wird einerseits einen 


\section{Berufliche Auswirkungen von Auslandspraktika}

Fragebogen ausfüllen und wird andererseits zu ihrer Berufsbiographie interviewt. Die Antworten auf den Fragebögen werden in ihrer Häufigkeit und Verteilung analysiert, die Antworten in den Interviews werden interpretiert und miteinander verglichen. In weiterer Folge werden die Aussagen aus den Interviews auch quantifiziert und nach ihrer Häufigkeit analysiert. Durch die ergänzenden Interviews kann der Kontext von Antworten aus den Fragebogendaten weiter erschlossen werden (Flick, 2016, S.42ff).

Über den Fragebogen werden in erster Linie die biographischen Daten der Person sowie Fakten über Schulausbildung, absolviertes Auslandspraktikum und die Berufsbiographie ermittelt. Erhoben werden vor allem folgende Daten: demographische Daten, besuchte Schule, Alter bei Beginn des Auslandspraktikums, Dauer des Auslandspraktikums, Fremdsprachenkenntnisse, die während des Praktikums als Arbeitssprache verwendete Fremdsprache, Praktikumsort, Sozialstatus der Personen gemessen an Ausbildung, Beruf und Einkommen der Eltern, berufliche Entwicklung und Laufbahn nach dem Schulabschluss, derzeitige Position und Aufgabenbereich im Unternehmen, derzeitiges Gehalt etc.

Die Befragung ist in Form eines halbstrukturierten Interviews geplant, das durch einen Interviewleitfaden eine grobe Struktur erhält und in einem narrativen Interviewteil den befragten Personen die Möglichkeit gibt, in Erzählform über ihre Schulausbildung, das absolvierte Auslandspraktikum und dessen Auswirkung auf die berufliche Entwicklung zu berichten (öffnende, erzählgenerierende Fragen und strukturierendes Nachfragen werden kombiniert). In der qualitativen Befragung werden unter anderem Fragen zu folgenden Bereichen gestellt: Motivation für ein Auslandspraktikum bzw. für die Wahl eines bestimmten Landes, Erwartungen vor dem Praktikum, Erfahrungen während des Praktikums, Auswirkungen des Auslandspraktikums auf die nachfolgende Schulzeit, Auswirkungen des Auslandspraktikums auf Berufswahl bzw. Studium, Auswirkungen des Auslandspraktikums auf die Berufslaufbahn etc.

Das Stichprobenverfahren ist eine Teilerhebung, d. h. es soll eine konkrete Auswahl von relevanten Personen befragt werden. Auf Basis der daraus abgeleiteten Aussagen wird eine Aussage über die Grundgesamtheit abgeleitet, daher ist es wichtig, dass die untersuchte Teilmenge für die Grundgesamtheit repräsentativ ist. Die Verteilung zuvor festgelegter Merkmale muss mit der Grundgesamtheit übereinstimmen. Um wissenschaftlich zulässige Aussagen zu treffen, die Wirksamkeit eines Auslandspraktikums zu messen zu und die Hypothesen beantworten zu können, wird eine Vergleichsgruppe mit Personen, die ihr Pflichtpraktikum in Österreich absolviert haben, befragt. Der Umfang der Stichprobe ist zum derzeitigen Zeitpunkt noch nicht festgelegt.

Der Fragebogen sowie der Interviewleitfaden werden in einem Pretest geprüft, um zu klären, ob die Fragen klar und verständlich sind und ob das Interview den geplanten Zeitrahmen nicht überschreitet. 


\section{| Berufliche Auswirkungen von Auslandspraktika}

Die Durchführung der Datenerhebung ist für das erste Halbjahr 2017 geplant. Bis dahin sollen die Vorarbeiten abgeschlossen und eine konkrete Stichprobenauswahl getroffen worden sein. Zuerst werden die ausgewählten Personen schriftlich mittels Fragebogen befragt, im Anschluss daran interviewt. Die Fragebogendaten werden statistisch erfasst und analysiert. Die Interviews werden transkribiert, analysiert und interpretiert, Zusammenhänge werden untersucht.

\section{Anmerkungen}

1 Berufsbildende mittlere und höhere Schulen mit Pflichtpraktikum: Technische, gewerbliche und kunstgewerbliche Schulen, Handelsakademien und Handelsschulen, Schulen für Mode und Bekleidungstechnik sowie für künstlerische Gestaltung, Schulen für Tourismus, Schulen für wirtschaftliche Berufe, Schulen für Sozialberufe, Land- und forstwirtschaftliche Schulen, Bildungsanstalten für Sozialpädagogik, Bildungsanstalten für Kindergartenpädagogik.

2 Höhere Lehranstalten für wirtschaftliche Berufe.

3 Höhere Lehranstalten für Tourismus.

4 Siehe Durchführung von Erasmus+ Mobilitätsprojekten (Bildung Erasmus+).

5 Nationalagentur für Lebenslanges Lernen (http://www.lebenslanges-lernen.at) ist Teil der OeAD (Österreichische Austauschdienst)-GmbH mit Sitz in Wien.

6 Einsetzbarkeit im Beruf bzw. Fähigkeit, auf dem Arbeitsmarkt zu bestehen.

7 European Credit System for Vocational Education and Training.

\section{Literatur}

Arbeiterkammer - Kammer für Arbeiter und Angestellte für Oberösterreich. (Hrsg). (2014). Pflichtpraktikum: Rechtliche Bestimmungen und Informationen. https://media.arbeiterkammer.at/ooe/publikationen/arbeitundrecht/B_2014_Pflic htpraktikum.pdf

Bader, R. (2000). Konstruieren von Lernfeldern - eine Handreichung für Rahmenlehrplanausschüsse und Bildungsgangkonferenzen in technischen Berufsfeldern. In R. Bader \& P. Sloane (Hrsg.), Lernen in Lernfeldern. Theoretische Analysen und Gestaltungsansätze zum Lernfeldkonzept. Markt Schaben: Eusl.

Bundesministerium für Bildung. (o. J.). Berufsbildende Schulen in Österreich. Lehrpläne. http://www.abc.berufsbildendeschulen.at/de/dlcollection.asp

Diekmann, A. (2016). Empirische Sozialforschung: Grundlagen, Methoden, Anwendungen. (10. Aufl.). Reinbek bei Hamburg: Rowohlt.

Drees, G. \& Pätzold, G. (2006). Praxisreflexion - Begriff und Gegenstand. In D. Euler (Hrsg.), Facetten des beruflichen Lernens. Bern: h.e.p. 


\section{Berufliche Auswirkungen von Auslandspraktika}

ECVET. (o. J.). European Credit System for Vocational Education and Training. http://www.ecvet-info.at/

ECVET projects. (2013). Template for Learning Agreement. Abgerufen am 18.08.2016 von

http://www.ecvet-projects.eu/toolbox/ToolBoxList.aspx?id=17\&type=1

Erasmus+. (o. J.). Erasmus+ Das Programm der Europäischen Union für Bildung, Jugend und Sport. Abgerufen am 18.08.2016 von http://www.erasmusplus.at/

Endres, W., Wiedenhorn T. \& Engel, A. (Hrsg.). (2008). Das Portfolio in der Unterrichtspraxis. Präsentations-, Lernweg- und Bewerbungsportfolio. Weinheim und Basel: Beltz

European Union (2014). The Erasmus Impact Study. http://ec.europa.eu/education/library/study/2014/erasmus-impact_en.pdf

Flick, U. (2016). Qualitative Sozialforschung. Eine Einführung. (7. Aufl.). Reinbek bei Hamburg: Rowohlt.

Gruber, H. (1999). Erfahrung als Grundlage kompetenten Handelns. Bern: Hans Huber.

Janson, K., Schomburg, H. \& Teichler, U. (2009). The Professional Value of Erasmus Mobility. The Impact of International Experience on Former Students' and on Teachers' Careers. http://www.acasecretariat.be/fileadmin/aca_docs/images/ members/2009_The_Professional_Value_of_ERASMUS_Mobility_01.pdf

JKU Linz. (o. J.). Abteilung für Pädagogik \& Pädagogische Psychologie. Forschungsgebiete. http://paedpsych.jku.at/PPP-Forschung/?page_id=129

Jones, E. (2012). Students perspecitves on going international. http://go.international.ac.uk/sites/default/files/Literature\%20Review_Student $\% 2$ 0Perspectives\%20on\%20Going\%20International.pdf

Loiselle, J. (1999). Interkulturelle Handlungskompetenz. In R. Huisinga, I. Lisop \& H.-D. Speier (Hrsg.), Lernfeldorientierung - Konstruktion und Unterrichtspraxis (S. 409-445). Frankfurt: Gesellschaft zur Förderung arbeitsorient. Forsch. u. Bild.

NEC Österreich. (o. J.). Nationales Europass Zentrum Österreich. http://www.europass.at/

Schwadorf, H. (2003). Berufliche Handlungskompetenz. Eine theoretische Klärung und empirische Analyse in der dualen kaufmännischen Erstausbildung. Stuttgart: Hohenheimer Schriftenreihe zur Berufs- und Wirtschaftspädagogik. 


\section{Berufliche Auswirkungen von Auslandspraktika}

\section{Verfasserin}

$\operatorname{Prof}^{\mathrm{in}} . \mathrm{Mag}^{\mathrm{a}}$. Karin Johanna Lindner, MAS MSc

Pädagogische Hochschule Oberösterreich

Institut Berufspädagogik, Studiengangskoordination Ernährung

Kaplanhofstraße 40

A-4020 Linz

E-Mail: karin.lindner@ph-ooe.at

Internet: http://www.ph-ooe.at/ 